\title{
The sGC stimulator BAY 41-8543 in a rat model of hypertension-induced heart failure
}

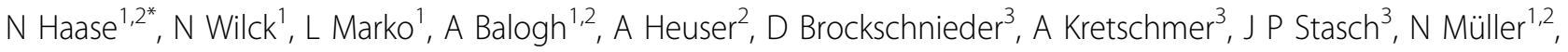 \\ $\mathrm{R}$ Dechend ${ }^{1,4}$
}

\begin{abstract}
From 7th International Conference on cGMP Generators, Effectors and Therapeutic Implications Trier, Germany. 19-21 June 2015
\end{abstract}

Hypertension with left ventricular hypertrophy is a major cause of diastolic heart failure (DHF). Due to its high prevalence and high rate of mortality, DHF represents a major challenge in today's cardiovascular medicine; with limited therapeutic options. Soluble guanylate cyclase (sGC) stimulation is emerging as a promising treatment option in DHF, and is currently under investigation in preclinical and clinical studies. The present study investigates the effect of the sGC stimulator BAY 41-8543 in a transgenic rat model of hypertension-induced heart failure.

We used 4 week-old male double transgenic rats expression both human renin and angiotensinogen genes (dTGRs). At 7 weeks of age, dTGRs exhibit striking cardiac hypertrophy with fibrosis and inflammation, ventricular arrhythmias and heart failure, which is accompanied with high mortality. We compared vehicle-treated dTGR (receiving 10\% transcutol, 20\% cremophor, $70 \%$ water) to those receiving $3 \mathrm{mg} / \mathrm{kg} / \mathrm{d}$ BAY 41-8543, and vehicletreated SD control rats (single oral dose per day for 3 weeks). We performed in vivo echocardiography, hemodynamic monitoring, cardiac electrophysiology studies and blood pressure measurements. Endothelial function was measured in isolated mesenteric arteries. Transcriptional analyses in cardiac tissue were performed using qRT-PCR and gene-microarray. Cardiac tissue was analyzed using histology.

Treatment of dTGRs with BAY 41-8543 resulted in $100 \%$ survival at week 7 , whereas only $24 \%$ of vehicletreated dTGRs survived. Mean arterial pressure in dTGRs was significantly by BAY 41-8543 reduced $(197 \pm 11 \mathrm{mmHg}$ vehicle vs $133 \pm 4 \mathrm{mmHg}$ BAY 41-8543). In addition, BAY 41-8543 significantly

\footnotetext{
* Correspondence: nadine.haase@mdc-berlin.de

'Experimental and Clinical Research Ctr., Max-Delbrueck Ctr. \& Medical Faculty of Charite, Berlin, Germany

Full list of author information is available at the end of the article
}

decreased in vivo total peripheral resistance and improved endothelium-dependent vasorelaxation of isolated mesenteric arteries. Furthermore BAY 41-8543 prevented fibrosis and inflammation of cardiac tissue. Echocardiography and invasive hemodynamic monitoring revealed BAY 41-8543 significantly increased ejection fraction and cardiac output in dTGR, whereas vehicle-treated had preserved systolic function but reduced diastolic function. In addition, diastolic compliance was significantly enhanced by BAY $41-8543$, as shown by myocardial strain analysis and end-diastolic pressure volume relationship (EDPVR); indicative of an improved diastolic function. In vivo programmed electrical stimulation revealed a high ventricular tachycardia induction rate in vehicle-treated dTGRs (46\%), which was significantly reduced in BAY 41-8543-treated dTGR (11\%). Myocardial gene-microarray analysis showed a reversal of dysregulated genes in dTGR by BAY 41-8543 treatment.

Our data demonstrate that BAY 41-8543 improves survival and cardiac performance in a transgenic rat model of hypertension-induced DHF. We postulate that treatment of DHF with sGC stimulators offers a novel therapeutic potential for humans.

\section{Authors' details}

${ }^{1}$ Experimental and Clinical Research Ctr., Max-Delbrueck Ctr. \& Medical Faculty of Charite, Berlin, Germany. ${ }^{2}$ Max-Delbrueck Ctr., Berlin, Germany. ${ }^{3}$ Bayer HealthCare, Global Drug Discovery, Wuppertal, Germany. ${ }^{4}$ Helios Klinik, Berlin, Germany.

Published: 2 September 2015

doi:10.1186/2050-6511-16-S1-A57

Cite this article as: Haase et al:: The sGC stimulator BAY 41-8543 in a rat model of hypertension-induced heart failure. BMC Pharmacology and Toxicology 2015 16(Suppl 1):A57. 